\title{
Excess properties of acetonitrile + methanol binary mixtures at microwave frequency
}

\author{
${ }^{1}$ A.P. Jogdand , ${ }^{2}$ Dr. P.L. Kadam \\ ${ }^{1}$ Physics research laboratory, Yeshwant Mahavidyalaya, Nanded-431605, Maharashtra, India \\ ${ }^{2}$ Physics research laboratory, Yeshwant Mahavidyalaya, Nanded-431605, Maharashtra, India
}

\begin{abstract}
Values of dielectric constant $\left(\epsilon^{\prime}\right)$ and dielectric loss $(\in ")$ have been experimentally determined for binary liquid mixtures of acetonitrile + methanol at $10.75 \mathrm{GHz}$ microwave frequencies at $30^{\circ} \mathrm{C}$. The values of $\in^{\prime}$ and $\in$ " have been used to evaluate the loss tangent $(\tan \delta)$, molar polarization $\left(\mathrm{P}_{12}\right)$, apparent polarization $\left(\mathrm{P}_{2}\right)$, the excess permittivity $\left(\Delta \in^{\prime}\right)$, the excess dielectric loss $\left(\Delta \in^{\prime \prime}\right)$ and excess molar polarization $\left(\Delta P_{12}\right)$. The calculated values of excess dielectric constant $\left(\Delta \in^{\prime}\right)$, excess molar polarization ( $\left.\Delta P_{12}\right)$ suggest that the structural behavior and dipolar rotation of the binary liquid mixtures. From the studied system indicates that the solute-solvent type of interaction taking place.

Density, viscosity and refractive index measurement of binary liquid mixtures were carried out at $30^{\circ} \mathrm{C}$. The values of viscosity have been used to evaluate the activation energy (Ea). The surface tension has been experimentally determined for pure liquid and binary liquid mixtures at $30^{\circ} \mathrm{C}$.In this paper we also report the range of physical properties includes density, refractive index, viscosity and surface tension.

The excess refractive index $\left(\Delta n_{D}\right)$, Excess square of refractive index $\left(\Delta n_{D}^{2}\right)$ and Excess value of apparent viscosity $(\delta \eta)$ of the viscous flow have also been estimated. These parameters have been used to explain the formation of 1:1 complex in the system.
\end{abstract}

Keywords: - Binary mixture, Dielectric parameters, Excess parameter, Molecular interaction

\section{INTRODUCTION}

Alcohols are industrially and scientifically important organic compounds and their physical and chemical properties are largely determined by $-\mathrm{OH}$ group alcohols are strongly associated in solutions because of dipole-dipole interaction and hydrogen bonding ${ }^{2}$.

Methanol is used as solvent and fuel due to synthetic and industrial applications. Therefore it seemed important to examine the dielectric behavior and excess properties of methanol with acetonitrile. In the earlier work we have reported the dielectric behavior of Acetonitrile + Methanol binary mixtures at microwave frequency ${ }^{1}$. In the present work we have been reported the excess properties of Acetonitrile + Methanol binary mixtures at microwave frequency.

A dielectric investigation of solutions containing varying amounts of interacting molecules helps to detect the formation and composition of complexes in them ${ }^{3}$. A survey of the literature shows that a few workers have tried to investigate some binary systems taking nitriles as one of the constituent components in the binary mixtures. Though the information in this field is steadily being enlarged by a number of workers ${ }^{4-8}$. The nature of complex formation in binary mixtures is still far from clear. With this in view, from experimental results the dielectric constant $\left(\in\right.$ ), dielectric loss $(\in$ ) $)$, loss tangent $(\tan \delta)$, molar polarization $\left(\mathrm{P}_{12}\right)$, apparent polarization $\left(\mathrm{P}_{2}\right)$, activation energy $(\mathrm{Ea})$, excess dielectric constant $\left(\Delta \in^{\prime}\right)$, excess dielectric loss ( $\Delta \in "$ ), excess molar polarization $\left(\Delta P_{12}\right)$, Excess activation energy ( $\Delta E a$ ), Excess loss tangent $(\Delta \tan \delta$ ), excess value of apparent viscosity $(\delta \eta)$ and excess square of refractive index $\left(\Delta n_{D}^{2}\right)$ have been calculated. Excess dielectric constant which can give information about the structural properties of polar liquids.

The studies of excess properties of (acetonitrile + methanol) binary liquid mixtures using frequency domain reflectometry (FDR) have not been carried out in the past.

Hence, we felt that the present investigation which may provide useful information about the formation of complexes in the acetonitrile + methanol binary liquid mixtures at $30^{\circ} \mathrm{C}$. 


\section{EXPERIMENTAL DETAILS}

The dielectric constant $\left(\epsilon^{\prime}\right)$ and dielectric loss $(\in ")$ were measured using Surber's technique ${ }^{9,}{ }^{10}$ of measuring the reflection coefficient from the air-dielectric boundary of the liquid in the microwave X-band at $10.75 \mathrm{GHz}$ frequency and at $30^{\circ} \mathrm{C}$ temperature.

The experimental setup is shown in figure 1 . The dielectric closed cell has a movable short. To hold the liquid in the cell, a thin mica window, whose VSWR and attenuation were neglected, was introduced between the cell and the rest of the microwave bench. Here source of reflex klystron 2 K 25 (USSR) was used.

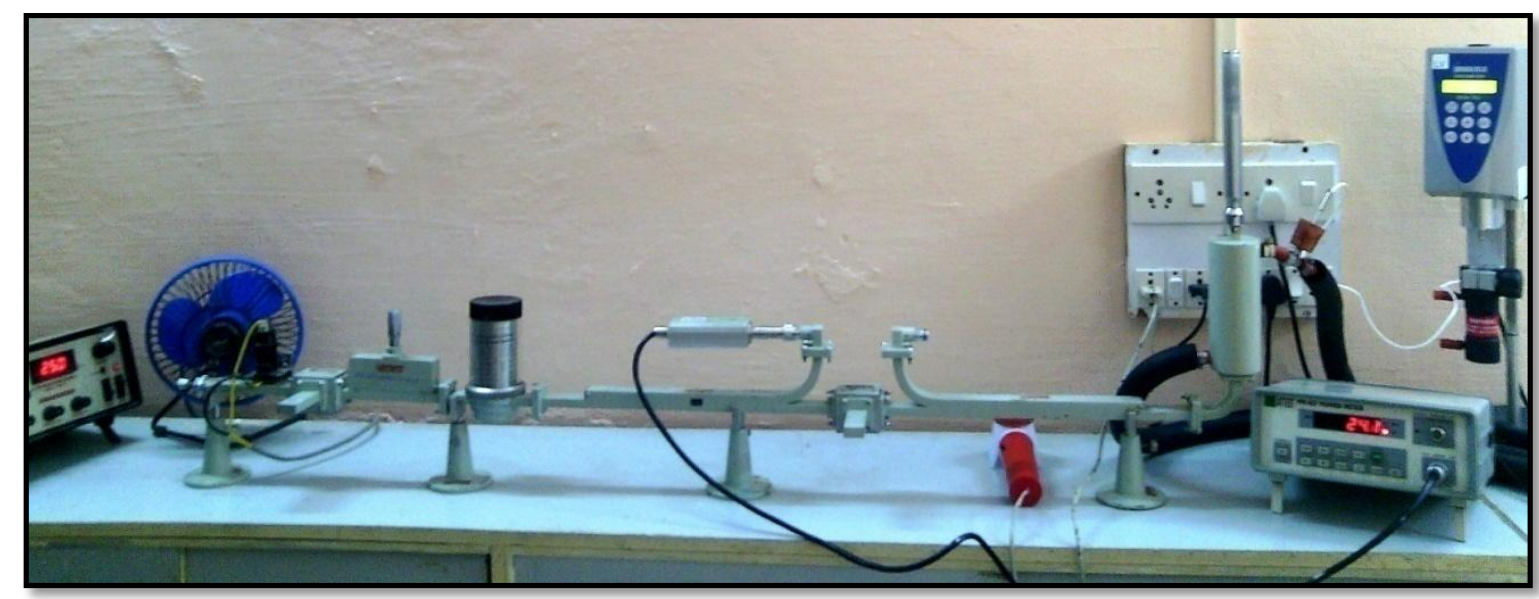

Figure 1. The experimental setup of microwave $\mathrm{X}$-band bench for the measurement of $\epsilon^{\prime}$ and $\in "$

A plunger wave guide is converted into a cavity by introducing a coupling hole in the entrance and shorting the other end with the calibrated plunger. The sample occupies the entire volume of the cavity the frequency is kept constant and the length of the plunger cavity is changed. Hence, several nodes appear as one increase the length of the cavity plunger, whenever the length of the cavity equals the half integral multiples of the guide wave length inside the medium. The plunger wave guide resonates the distance through which the plunger is moved between the successive cavity nodes gives half of the wave length $(\lambda d)$ of the microwave inside the medium.

The measurement of reflected power at resonance gives the attenuation coefficient of the sample $^{11}$.Surber has derived the following relations for the dielectric parameters $\in$ ', $\in$ "

$$
\begin{aligned}
& \in^{\prime}=\left(\frac{\lambda_{0}}{\lambda_{c}}\right)^{2}+\left(\frac{\lambda_{0}}{\lambda_{d}}\right)^{2}\left[1-\tan ^{2}\left(\frac{1}{2} \tan ^{-1} D\right)\right] \\
& \epsilon^{\prime \prime}=\frac{1}{\pi}\left(\frac{\lambda_{0}}{\lambda_{d}}\right)^{2} \alpha_{d} \lambda_{d}
\end{aligned}
$$

Where $\mathrm{D}$ is the dissipation factor, $\alpha_{d}$ is the attenuation constant due to dielectric, $\lambda_{d}$ is the wave length of the e.m. wave in the wave guide filled with the dielectric $\lambda_{0}$ is the free space wavelength, $\lambda_{c}=2 a$ is the cutoff wavelength for the wave guide. $\alpha_{d} \lambda_{d}$ is the attenuation per wavelength. Having determined $\alpha_{d} \lambda_{d}, \lambda_{0} \lambda_{c}$ and $\lambda_{d}$ the values of $\in{ }^{\prime}, \in$ " may be calculated by using the equations (1) and (2) respectively.

The density of pure components and their mixtures were measured by using DMA 35 portable vibrating density meter, AntonPaar, Austria (Europe). The parts of enhanced ULA adapter : ULA-49 EAY water jacket, sample chamber, tube end cap ULA-34, ULA-31 EY, ULA-31 EYZ, clamping collar ULA-OZE of Brook field engineering laboratories USA and low temperature circulating water bath, Nivtech Instruments \& Engineers, Thane, India at $30^{\circ} \mathrm{C}$. Specification of density meter, AntonPaar Austria is accuracy $0.001 \mathrm{~g} / \mathrm{cm}^{3}$ measuring range density 0 to $3 \mathrm{~g} / \mathrm{cm}^{3}$. Temperatures 0 to $40^{\circ} \mathrm{C}$.

Viscosity of pure components and their mixtures were measured by using viscometer Brook field DVII + Pro model LVDV - II + P Brook field engineering laboratories, INC, USA, calibration of this instrument will be accurate to within $\pm 1 \%$ of its full scale range.

The refractive index of the pure components and their mixtures were measured by using Abbe's refractometer (with Glass scale) Mittal Enterprises, New Delhi, India, having an accuracy 0.001 by reading and 0.0001 by estimation. Measuring range extends from 1.300 to 1.700 with the help of sodium D line surface 
tension of pure components and their mixtures were measured by using Stalagmometer at room temperature $30^{\circ} \mathrm{C}$.

Acetonitrile (AR grade) purity (GC) $\leq 10$. Identity IR supplied by Merck KGaA, Darn Stadt, Germany and methanol (AR Grade) methanol laboratory Reagent $>=99.6 \%$ (GC) Supplied by sigma-Aldrich Steinheim Germany were used without further purification.

The solutions were prepared by mixing acetonitrile + methanol in volume. These binary liquid mixtures according to their proportions were mixed well and kept for 6 hours in a well stoppered bottle to ensure good thermal equilibrium. Microwave input and output power measured by Pm-437 (Attest) power meter, Chennai, India. Rectangular wave guide working $\Gamma_{E 10}$ mode $10 \mathrm{~dB}$, VidyutyantraUdyog, India.

Low temperature water circulating both was used for maintaining temperature of pure components and their binary liquid mixtures for measurement of viscosity, refractive index, density and plunger reading using Xband microwave bench.

\section{RESULTS AND DISCUSSION}

Dielectric constant $\left(\in^{\prime}\right)$, dielectric loss $\left(\in \in^{\prime}\right)$, loss tangent $(\tan \delta)$, molar polarization $\left(p_{12}\right)$, apparent polarization $\left(\mathrm{P}_{2}\right)$ and activation energy $\left(E_{a}\right)$ for the viscous flow with increasing mole fraction $\left(\mathrm{X}_{\mathrm{A}}\right)$ of Acetonitrile for the binary mixture Acetonitrile + Methanol are listed in Table 1.The values of viscosity $(\eta)$, refractive index $\left(n_{D}\right)$, density $(\rho)$, surface tension $(\mathrm{T})$, square of refractive index $\left(n_{D}^{2}\right)$, mole fraction of solute and solvent are listed in Table 2

\section{Excess Parameters}

The excess values of permittivity $\Delta \in^{\prime}, \Delta \in^{\prime \prime}$, excess molar polarization $\Delta P_{12}$, excess refractive index $\Delta \eta_{D}$, Excess square of refractive index $\Delta \eta_{D}^{2}$, Excess activation energy ( $\Delta E a$ ), apparent excess value of viscosity ( $\delta \eta$ ), Excess loss tangent ( $\Delta \tan \delta$ ), for the system acetonitrile + methanol are reported in figure 2 to 9 and listed in Table 3. The excess values were then calculated by using the relations of the form

$$
\Delta Y=Y_{m}-\left(X_{A} Y_{1}+X_{B} Y_{2}\right)
$$

Where $\Delta Y$ any excess parameter and $\mathrm{Y}$ refers to the above mentioned quantities. The subscripts $\mathrm{m}, 1$ and 2 used in the above equation are respectively for the mixture, component 1 and $2, X_{A}$ and $X_{B}$ are the mole fractions of the two components in the liquid mixtures. Mole fraction represented by mole/L.

Activation energy (Ea) of the viscous flow for the pure liquid and their liquid mixtures is obtained by using the relation.

$$
E a=2.303 \frac{R T}{J} \log _{10}\left(\frac{\eta V}{N h}\right)
$$

Where $\eta$ and $\mathrm{V}$ are the viscosity and the molar volume of the liquids respectively and other symbols have their usual meaning.

Table No.1. Mole Fraction of Solute $\left(\mathbf{X}_{\mathrm{A}}\right)$, Dielectric Constant $\left(\in \in^{\prime}\right)$, Dielectric loss $(\in$ "), Loss Tangent ( $\tan \delta$ ), Molar Polarization $\left(\mathbf{P}_{12}\right)$, apparent Polarization $\left(\mathbf{P}_{2}\right)$ and Activation Energy (Ea) of Binary Liquid Mixtures at $30^{\circ} \mathrm{C}$

\begin{tabular}{|c|c|c|c|c|c|c|c|}
\hline $\begin{array}{c}\text { Sr. } \\
\text { no. }\end{array}$ & $\mathbf{X}_{\mathbf{A}}$ & $\in^{\prime}$ & $\in^{\prime \prime}$ & $\tan \delta$ & $\mathbf{P}_{\mathbf{1 2}}$ & $\mathbf{P}_{\mathbf{2}}$ & $\mathbf{E a}$ \\
\hline 1 & 0 & 7.705522 & 4.335968 & 0.562709 & 28.307365 & 0 & 5.690382 \\
\hline 2 & 0.078505 & 11.225008 & 5.039032 & 0.448911 & 32.414324 & 80.621982 & 5.65193 \\
\hline 3 & 0.160852 & 13.645505 & 5.401292 & 0.395829 & 34.666704 & 67.842708 & 5.610854 \\
\hline 4 & 0.247329 & 16.788696 & 6.122681 & 0.364691 & 36.93315 & 63.183118 & 5.589215 \\
\hline 5 & 0.338256 & 19.248264 & 5.894131 & 0.306216 & 38.70742 & 59.053471 & 5.574344 \\
\hline 6 & 0.433985 & 22.622302 & 6.156992 & 0.272165 & 40.625408 & 56.690934 & 5.566769 \\
\hline 7 & 0.534907 & 23.193409 & 5.764088 & 0.248523 & 41.886387 & 53.693127 & 5.559097 \\
\hline 8 & 0.641456 & 27.624985 & 5.251968 & 0.190117 & 43.902986 & 52.620211 & 5.551327 \\
\hline 9 & 0.754116 & 29.222987 & 5.006236 & 0.171312 & 45.476408 & 51.074476 & 5.543454 \\
\hline 10 & 0.873428 & 29.80699 & 4.887068 & 0.163957 & 46.887221 & 49.579703 & 5.535478 \\
\hline 11 & 1 & 29.93533 & 4.595124 & 0.153502 & 48.416779 & 48.416779 & 5.527394 \\
\hline
\end{tabular}


Table:2. Mole fraction of solute $\left(\mathbf{X}_{\mathrm{A}}\right)$, Mole Fraction of Solvent $\left(\mathbf{X}_{\mathbf{B}}\right)$, Density $(\rho)$

Viscosity $(\eta)$, Refractive Index $\left(n_{D}\right)$, Square of Refractive Index $\left(\left(n_{D}\right)^{2}\right)$ and Surface Tension $(T)$ of Binary

Liquid Mixtures at $30^{\circ} \mathrm{C}$

\begin{tabular}{|c|c|c|c|c|c|c|c|}
\hline Sr.no. & $\mathbf{X}_{\mathbf{A}}$ & $\mathbf{X}_{\mathbf{B}}$ & $\begin{array}{c}\rho \\
\mathbf{g m} / \mathbf{c m}^{\mathbf{3}}\end{array}$ & $\begin{array}{c}\eta \\
\mathbf{c P}\end{array}$ & $\mathbf{n}_{\mathbf{D}}$ & $\left(\mathbf{n}_{\mathbf{D}}\right)^{\mathbf{2}}$ & $\begin{array}{c}\mathbf{T} \\
\mathbf{d y n e} / \mathbf{c m}\end{array}$ \\
\hline 1 & 0 & 1 & 0.782 & 0.97 & 1.331 & 1.771561 & 18.824231 \\
\hline 2 & 0.078505 & 0.921495 & 0.7811 & 0.91 & 1.332 & 1.774224 & 20.306787 \\
\hline 3 & 0.160852 & 0.839148 & 0.7808 & 0.85 & 1.333 & 1.776889 & 21.034937 \\
\hline 4 & 0.247329 & 0.752671 & 0.7797 & 0.82 & 1.334 & 1.779556 & 21.678919 \\
\hline 5 & 0.338256 & 0.661744 & 0.7785 & 0.8 & 1.335 & 1.782225 & 22.692413 \\
\hline 6 & 0.433985 & 0.566015 & 0.7771 & 0.79 & 1.336 & 1.784896 & 23.59542 \\
\hline 7 & 0.534907 & 0.465093 & 0.7752 & 0.78 & 1.337 & 1.787569 & 24.425945 \\
\hline 8 & 0.641456 & 0.358544 & 0.7742 & 0.77 & 1.338 & 1.790244 & 25.356261 \\
\hline 9 & 0.754116 & 0.245884 & 0.7719 & 0.76 & 1.339 & 1.792921 & 25.621151 \\
\hline 10 & 0.873428 & 0.126572 & 0.7709 & 0.75 & 1.34 & 1.7956 & 26.57846 \\
\hline 11 & 1 & 0 & 0.7682 & 0.74 & 1.341 & 1.798281 & 25.982485 \\
\hline
\end{tabular}

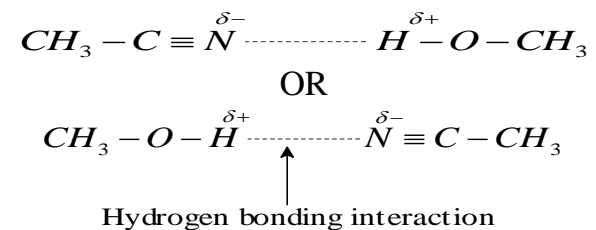

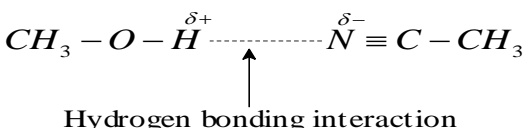

\subsection{Excess dielectric constant}

The values of excess dielectric constant shows that

(i) $\quad \Delta \in^{\prime}=0$ Indicates that mixtures constituents do not interact and thus have ideal mixing behavior;

(ii) $\Delta \in^{\prime}<0$ Indicates that mixture constituents interact so as to reduce the total number of effective dipoles that contributed to the mixture dielectric polarization.

(iii) $\Delta \in^{\prime}>0$ Indicates that the constituents of a mixture interact in such a way that there is an increase in number of effective dipoles contributed in the mixture dielectric polarization.

(iv) The magnitude of $\Delta \in^{\prime}$ values is the evidence of the strength of unlike molecules H-bond interactions; that is, higher $\Delta \in^{\prime}$ values represent the stronger H-bond unlike molecular connectivity's between unlike molecules and vice versa.

(v) The molar concentration corresponding to pronounced maximum of $\Delta \in^{\prime}$ values represents the stoichiometric ratio of a stable adduct in the mixture. [Jeevanandham P. et.al. ${ }^{12,13}$, Ch.V.V. Ramana et.al. ${ }^{4}$ 2013] The variation of $\Delta \in^{\prime}$ as a function of concentration is graphically represented in figure (2) observation of variation of $\Delta \in^{\prime}$ with mole fraction of acetonitrile in binary mixture indicates that maxima occurring at $0.433985,0.641456$ and minima occur at 0.534907 mole fraction of acetonitrile. It is positive and less in magnitude. The positive deviation from ideal behavior ( $\Delta \in^{\prime}$ being positive) is qualitatively attributed to a build in of components of the mixture in the structure of respective solvent. $\Delta \in^{\prime}>0$ Indicate that the two solvents dipole moment increases. There is formation of multimers and dimmers. A dimer is a chemical or biological entity consisting of two structurally similar subunits called monometers, which are joined by bonds, which can be strong or weak. Generally a multimer implies a few monomers attached together. But polymer will be formed with many numbers of monomers. The excess dielectric constant which varies a function of concentration and reaches an optimum ratio at 0.641456 indicates the liquid mixtures in such a way that the total effective dipole moment increases. 


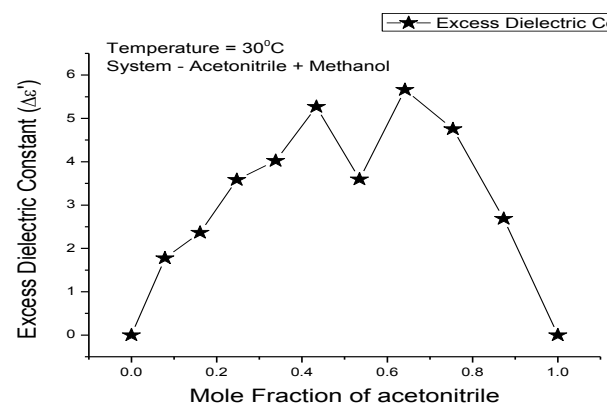

Figure2. Variation of Excess Dielectric

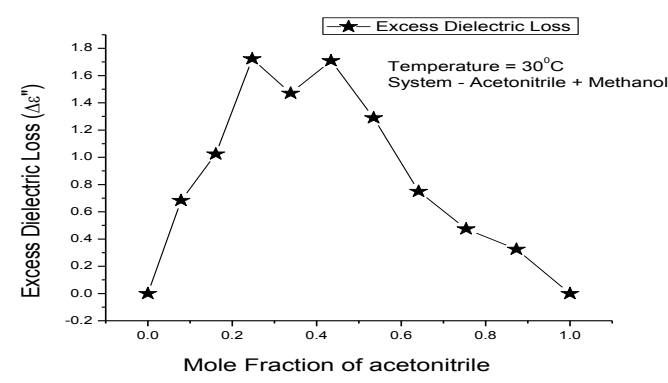

Figure3. Variation of Excess Dielectric Loss versus Mole fraction of acetonitrile in the mixture

Constant $\left(\Delta \in^{\prime}\right)$ versus Mole fraction of $\left(\Delta \in \epsilon^{\prime \prime}\right)$ Acetonitrile in the mixture

There is a tendency of dipole aligned in parallel direction. The nonzero $\Delta \in^{\prime}$ values of the concentration of mixtures figure (2) suggest that electron transport will be made easily from one molecule to other, because electron flow in a medium will increase as well as the interactions in between acetonitrile and methanol through hydrogen bonding interaction. We expect that excess dielectric properties depend on electron transport. Same behavior is obtained ${ }^{14,15}$.

\subsection{Excess molar polarization $\left(\Delta P_{12}\right)$}

$\Delta P_{12}$ has been often used to discuss the molecular interaction in non-electrolyte binary mixtures ${ }^{15}$. The molar polarization of substances that allows electron Polarizability of molecules in various states of aggregation (gaseous, liquid and solid) to be determined. It is necessary for calculating the dipole moment of the complex from the experimental data. Higher value in the system may be due to the effective of hydrogen bonding in a mixed solvent ${ }^{16}$.

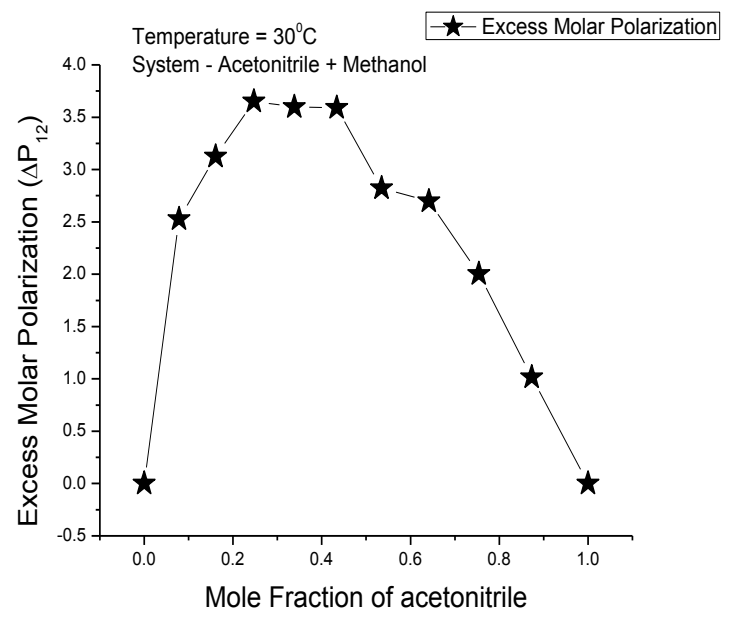

\section{Figure 4.Variation of Excess Molar Polarization $\left(\Delta P_{12}\right)$ versus Mole fraction of acetonitrile in the} mixture

The observed values of excess molar polarization in figure (4) are remains positive in associated rich region. This is must probably due to the fact that the parallel alignment of molecular dipoles is the dominant factor in the associated rich region where the long range electrostatic interaction plays a vital role for polarization. ${ }^{12,13,17}$ Same behavior is obtained. ${ }^{13,15,18}$ Unit of $\Delta P_{12}$ is $\mathrm{cm}^{3} \mathrm{~mol}^{-1}$.

\subsection{Excess refractive index $\left(\Delta n_{D}\right)$}

The values of $\Delta n_{D}$ versus mole fraction at $30^{\circ} \mathrm{C}$ presented in figure (5) are all positive for binary liquid mixtures of acetonitrile + methanol. Excess refractive indices shows that the solvent-solvent interactions in the case of proticprotic and aprotic-protic system is of a type of hydrogen bond formation as a result of the amphiprotic hydrogen bond acceptor donor (HBA-D) 
The maximum value of the refractive index indicates maximum solvent-solvent interaction and depends mainly on the different physical properties of the solvents such as the dielectric constant, dipole moment, donor number, chemical structure, the salvatochromic quantitative values of KamletTaft hydrogen bond acidity, basicity and diplarity - Polarization ${ }^{5}$.Similar behavior is obtained ${ }^{5,19,20}$.

Hence the values of $\Delta n_{D}$ are all positive due to the strong hydrogen bonding between acetonitrile and methanol.

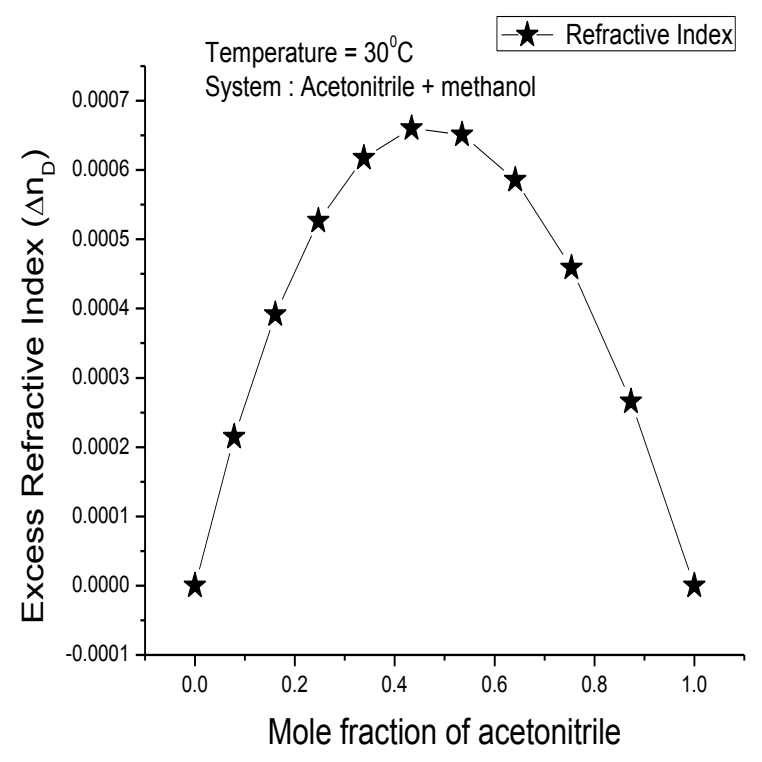

Figure5. Variation of Excess Refractive Index $\left(\Delta n_{D}\right)$ versus Mole fraction of acetonitrile In the mixture

1.4 Excess square of refractive index $\left(\Delta n_{(D)}^{2}\right)$

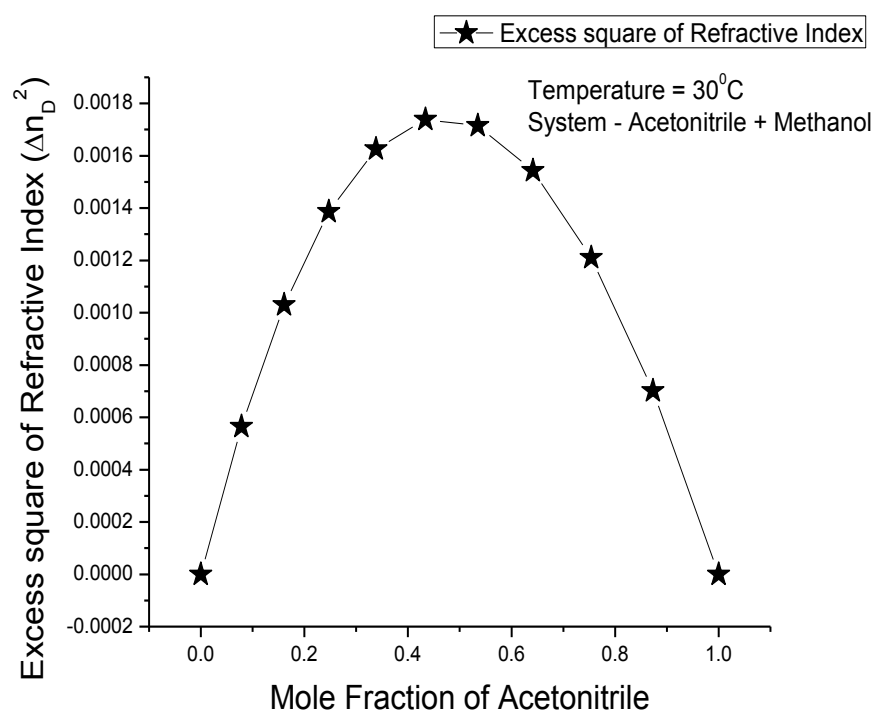

Figure6. Variation of Excess square of Refractive Index $\left(\Delta n_{D}^{2}\right)$ versus Mole fraction of acetonitrile in the mixture

Excess values of square of refractive index $\left(\Delta n_{(D)}^{2}\right)$ versus mole fraction presented in figure (6). Excess Square of refractive index values are all positive indicating strong interactions between acetonitrile + 
methanol molecules. For this excess square of refractive index the maxima for acetonitrile + methanol mixture occur at about 0.433985 mole fraction of acetonitrile ${ }^{10,21}$.

Excess Square of refractive index curve suggests the formation of 1:1 complex in the mixture of acetonitrile + methanol molecules.

Table No. 3. Mole Fraction of Solute $\left(\mathbf{X}_{\mathbf{A}}\right)$, Excess Molar polarization $\left(\Delta P_{12}\right)$, Excess dielectric constant ( $\left.\Delta \in^{\prime}\right)$, Excess dielectric Loss ( $\left.\Delta \in "\right)$, Apparent Excess value of Viscosity $\left(\delta_{n}\right)$, Excess square of Refractive index $\left(\Delta n_{D}^{2}\right)$, Excess Activation Energy $\left(\Delta E_{a}\right)$ and Excess loss tangent $(\Delta \tan \delta)$ at $30^{\circ} \mathrm{C}$

\begin{tabular}{|c|c|c|c|c|c|c|c|c|}
\hline $\begin{array}{c}\text { Sr. } \\
\text { No. }\end{array}$ & $\mathrm{X}_{\mathrm{A}}$ & $\Delta P_{12}$ & $\Delta \in^{\prime}$ & $\Delta \in "$ & $\begin{array}{c}\delta_{n} \\
\mathrm{cP}\end{array}$ & $\Delta n_{D}^{2}$ & $\Delta E_{a}$ & $\Delta \tan \delta$ \\
\hline 1 & 0 & 0 & 0 & 0 & 0 & 0 & 0 & 0 \\
\hline 2 & 0.078505 & 2.528269 & 1.774335 & 0.682719 & -0.041944 & 0.000565 & -0.025657 & -0.081673 \\
\hline 3 & 0.160852 & 3.1247 & 2.364274 & 1.023638 & -0.083004 & 0.00103 & -0.053311 & -0.101058 \\
\hline 4 & 0.247329 & 3.652144 & 3.585098 & 1.722616 & -0.093114 & 0.001386 & -0.060855 & -0.096809 \\
\hline 5 & 0.338256 & 3.597925 & 4.023376 & 1.470502 & -0.092201 & 0.001626 & -0.060906 & -0.118076 \\
\hline 6 & 0.433985 & 3.590859 & 5.269377 & 1.708554 & -0.080183 & 0.001739 & -0.052879 & -0.112954 \\
\hline 7 & 0.534907 & 2.822356 & 3.597007 & 1.289496 & -0.066971 & 0.001715 & -0.044102 & -0.095298 \\
\hline 8 & 0.641456 & 2.696317 & 5.660019 & 0.749763 & -0.052465 & 0.001543 & -0.034505 & -0.110104 \\
\hline 9 & 0.754116 & 2.004212 & 4.753611 & 0.474834 & -0.036553 & 0.00121 & -0.024016 & -0.082807 \\
\hline 10 & 0.873428 & 1.015731 & 2.685331 & 0.324746 & -0.019112 & 0.000701 & -0.012546 & -0.041339 \\
\hline 11 & 1 & 0 & 0 & 0 & 0 & 0 & 0 & 0 \\
\hline
\end{tabular}

\subsection{Excess activation energy $\left(\Delta E_{a}\right)$}

Figure (7) shows the excess activation energy $\left(\Delta E_{a}\right)$ for viscous flow versus mole fraction of acetonitrile at $30^{\circ} \mathrm{C}$ for the mixture of acetonitrile + methanol. The excess activation energy for viscous flow are negative at $30^{\circ} \mathrm{C}$ for the binary liquid mixture of acetonitrile + methanol. Same behavior is obtained ${ }^{22}$.

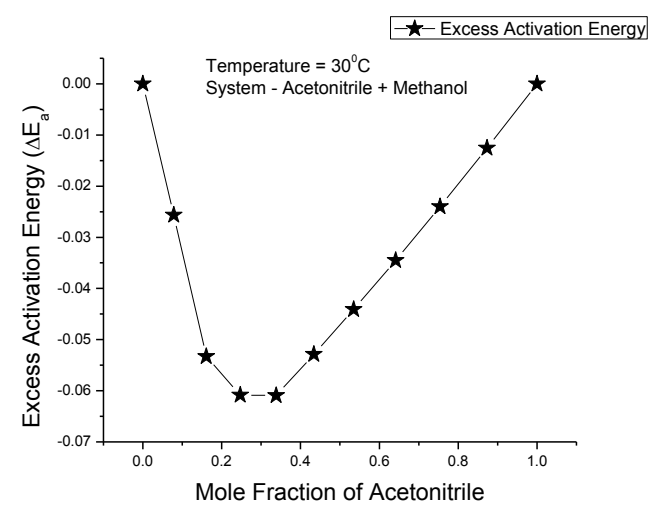

Figure7. Variation of Excess Activation Energy ( $\Delta E a$ ) versus Mole fraction of acetonitrile In the mixture

\subsection{Apparent excess value of viscosity $\left(\delta_{n}\right)$}

It is preferred to define equation (3) as the apparent excess values of viscosity $\delta_{\eta}$ instead of excess viscosity $\Delta \eta$ (Shantilaloswal et.al. ${ }^{18}$ ) as it does not represent true excess function as per the definitions of excess functions.

Values of $\delta_{\eta}$ are small and negative for the mixtures (23) of the system. The mixtures acetonitrile + methanol shows significant negative excess viscosity probably due to changes in the liquid associated structure of methanol $^{22}$.This behavior is characteristic of system in which at least one of the components exhibits hydrogen bonding. (MIGUEL-KATZ et.al. ${ }^{24}$ )Figure (8) shows Apparent Excess values of viscosities for acetonitrile + methanol system. Minimum value of $\delta_{n}$ at $\mathrm{X}_{\mathrm{A}}=0.247329$ in agreement with what has been said above and formation of hydrogen bonding in the system acetonitrile + methanol. Some behavior is obtained in the mixture of aniline + toluene, aniline + butanol ${ }^{25}$. 
Anwar Ali et.al. ${ }^{26}$, Ezekiel D.Dikio et.al. ${ }^{27,}{ }^{28}$ reported the negative $\Delta \eta$ values are generally observed for the system where dispersion of weak dipole-dipole forces is primarily responsible for the interactions between the component molecules. Negative deviation in $\Delta \eta$ may also be observed due to the difference in the molecular size of the component molecule ${ }^{26}$ as in the present mixtures. Same behavior is obtained ${ }^{18}$.

The strength of the specific forces is not the factor influencing the viscosity deviation in the liquid mixture. This leads to suggestions that combinations of an interactive and non-interactive force are responsible in positive and negative interactions. (Ezekiel D. Dikio et.al. ${ }^{27}$ )

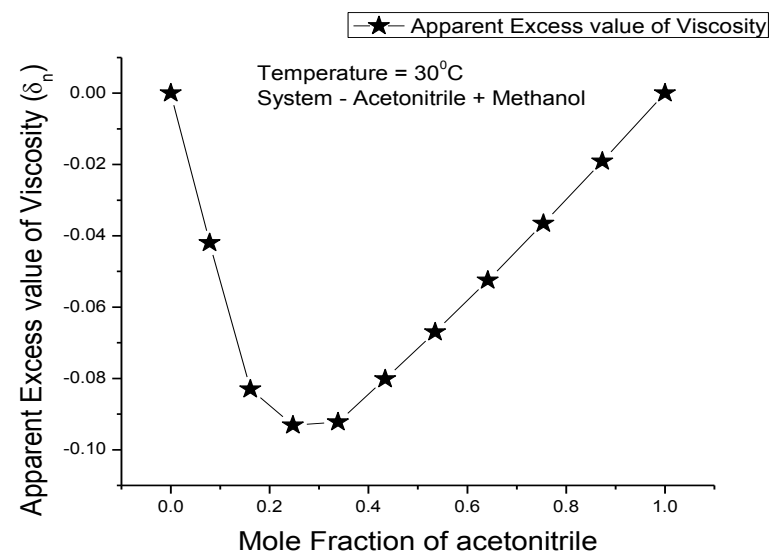

Figure 8. Variation of Apparent Excess value of Viscosity ( $\delta \eta)$ versus Mole fraction of acetonitrile in the mixture

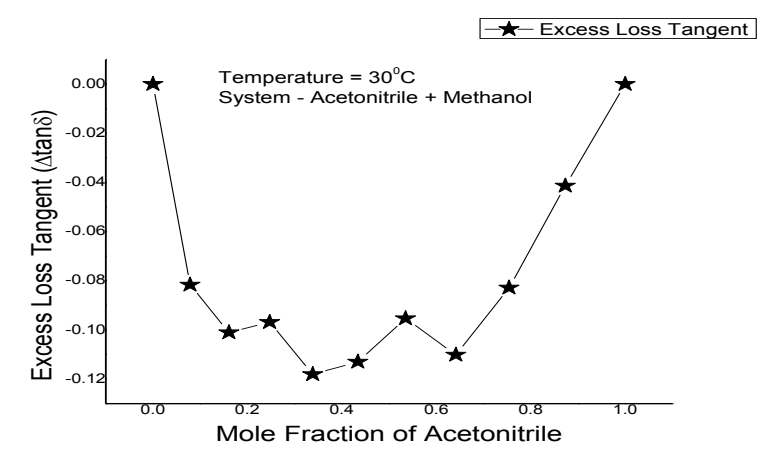

Figure9. Variation of Excess Loss Tangent ( $\Delta \tan \delta$ ) versus Mole fraction of acetonitrile In the mixture

\section{CONCLUSIONS}

Excess dielectric constant $\left(\Delta \in^{\prime}\right)$, Excess molar polarization $\left(\Delta P_{12}\right)$, Excess refractive index $\left(\Delta n_{D}\right)$, excess square of refractive index $\left(\Delta n_{D}^{2}\right)$, are all positive values indicating strong interactions between acetonitrile + methanol molecules.

There is intermolecular interaction among the components of the binary mixtures leading to hydrogen bond formation of the type 1:1 complex formation between acetonitrile + methanol mixtures.

\section{REFERENCES}

[1] A.P.Jogdand and Dr. P.L.Kadam, Dielectric behavior of Acetonitrile + Methanol binary mixtures at microwave frequency. IOSR Journal of Applied Physics , feb,2014 vol. 6issue:1(version-2) ,e-ISSN: 2278-4861 Pg. 14-22

[2] S.V. Kumara Sastry, S. SreehariSastry and VRK Murthy, Excess dielectric and thermodynamical studies on hydrogen bonded binary mixtures of propan-1-ol with methyl and ethyl benzoates, Chem. Sci. Trans. 2012, 1 (3), 638-652.

[3] RekhaPande\& G.M. Kalamse, Asian J. Chemistry Vol. 17, No. 1 (2005), 283-288.

[4] Ch. V.V. Ramana, A.B.V. Kirankumar, M. Ashok kumar and M.K. Moodley Dielectric and excess dielectric constant of acetonitrile + Butyl amine + Ethylamine and methylamine at 303, 313, and $323 \mathrm{~K}$

[5] Journal of chemistry volume 2013 (2013), Article ID 687106 
[6] Farid I. EI. Dossoki refractive index and density measurements for selected binary protic - protic, aprotic - aprotic, and aprotic-protic systems at temperatures from $298.15 \mathrm{~K}$ to $308.15 \mathrm{~K}$. J. of the Chinese chemical society 2007, 54, 1129-1137.

[7] Abeer K. Shams Densities, refractive indices and excess properties of binary mixtures of acetonitrile with Benzene, Toluene n-xylene and mesitylene at temperatures from (298.15 to $313.15 \mathrm{~K})$, Journal of Al. Nahrain University Vol. 14 (2), June, 2011, Pp. 75-85.

[8] R.K. Sukla, G.K. Gupta, S.K. Puranik, A.K. Sharma and Balwant Singh, surface tension of Binary liquid mixtures from various liquid state models at 293.15, 298.15 and 313.15 K, Int. J. of Latest Research in Science and Technology vol. I. Issue 3 Page NO. 276-281 Sept-Oct (2012)

[9] Ch. V.V. Ramana G. Ramachandra Reddy and M. Mohan Reddy, Dielectric and excess dielectric constant of acetonitrile + hexane + heptanes and + cyclohexane at 303-313 and 323 K, Physical chemistry, Vol. 4, No. 2, 2009.

[10] W.H. Surber, 1, Appl. Phys. 19, 514 (1948)

[11] P.J. Singh and K.S. Sharma Dielectric behaviour of ketone-amine binary mixtures at microwave frequencies pramana Journal of Physics Vol. 46, No.4, April 1996, Pp. 259-270.

[12] P. Job Ann. Chem. 9,113, (J1928)

[13] A. Gavezzotti; Molecular aggregation of acetic acid in a carbon tetra chloride solution : A molecular Dynamics study with a view to crystal Nucleation J. Chem. Eur. 5 (1999)

[14] Siddharthkamble, Y.S. Sudake P.W. Khirade and S.C. Mehrotra Dielectric constant, Density and R.I. Study on mixing properties of binary liquid mixtures, Int. J. of pharma and Bio. Sciences 2012 Oct.; 3 (4), (P) 382-391.

[15] SiddharthKamble, Y.S. Sudake, S.S. Patil, P.W. Khirade and S.C. Mehrotra, Dielectric and opticl properties of polar and non-polar liquids at different temperatures, Int. J. of Pharma and Bio-Sciences Vol. 2 / Issue 21 April - Jan 201.

[16] N.V. Shastry, S.R. Patel \& J. George, Excess thermodynamic and dielectric functions of the binary liquid mixtures of methylmethacrylate + alcoxyethanols (2-methoxyethanol, 2-ethoxyethanol, 2-butoxyethanol) and +1-alcohols (1-butanol, 1-pentanol, 1-heptanol), Indian Journal of Chemistry, Vol. 39 A, Dec. 2000, pp. $1270-1279$.

[17] B.B. Swain ActaChim. Hung 118, 321 (1985)

[18] R.L. Mishra J.D. Pandey, Indian Journal of Pure appli. Phys. 15, 505 (1977).

[19] ShantilalOswal, Studies on density, dielectric constant and Refractive Index of binary mixtures of esters in between and carbon tetra chloride, Can. J. Chem. 66, 111 (1988)

[20] UfukSancar, Vural, V. Muradoglu and SedatVaral, Excess molar volumes and Refractive Index of Binary mixtures of Glycerol + Methanol and Glycerol + water at 298.15 K and 303.15 K, Bull Chem. Soc. Gthjop, 2011, 25 (1) 111-118.

[21] Marion martinez-Reina, Eliseo Amado-Gonzalez, Refractive indices, Densities and excess properties of Binary mixtures of ethanol with Hexane, Heptanes, Octane and water at $(293.15,298.15,303.15$ and 308.15) K, Bitsu : Revistadelafaculted de CiencicaBasicas, Vol. 8, Num. 2, 2010, pp. 1-10, Universidad de Pamplona Pamplona, Colombia.

[22] B.S. Narwade, P.G. Gawali, RekhaPande and G.M. Kalamse, Dielectric studies of binary mixtures of npropyl alcohol and ethylenediamine J. Chem. Soci. Vol. 117, No. 6, Nov. 2005, Pp. 673-676.

[23] A mariano, A Camacho, M. Postiago, A. Valen, H. Artigas, F.M. Royo and J.S. Urienta, Branzillian Journal of Chemical Engineering Vol. 17, 4.4-7 Sau-Poulo Dec. 2000.

[24] Comb. L.L. Mc Mohan W.H. Farish S.H. J. Phy. Chem. 75 (1971), 2133.

[25] MIGUEL KATZ, Pedro W. Lobo, A Sanchominand and Horaciosolimo, Argentina, Canadian Journal of Chemistry 49, 2605 (1971)

[26] Mignel, Katz, Pedro, W. obo, A. Sancho minaco and HoracioSolim O, Canadian J. of Chemistry 49, 2605 (1971)

[27] Anwar Ali, Anil Kumar Nain, Dinesh Chand and Rizwan Ahmad, Viscosities and refractive indices of Binary mixtures of dimethyl sulphoxide with some Aromatic hydrocarbons at different temperatures. An experimenta and theoretical study J. of the Chinese Chemical Society, 2006, 53, 531-543.

[28] Ezekiel D. Dikio, Simphiwe M. Nelana, David A. Isabirye, E no E. Eberso, Density, Dynamic viscosity and derived properties of binary mixtures of methanol, Ethanol, n-propanal and n-Butanol with pyridine at $\mathrm{T}=(293.15,303.15$ and 323-15) K, Int. J. Electrochem. Sci. 7 (2012) 11101-11122.

[29] Jeevanandham P, Kumar S. Periyasamy, P. and Sedhumatharen K. Dielectric properties of binary liquid mixtures of aniline, o-chloroaniline and o-anisidine

[30] with carbon tetrachloride at 303.15K, Int. J. of Recent Scientific research Vo. 3 Issue 10 pp. 878-883, October 2012. 